\title{
ON COMMUTING FAMILIES OF NONEXPANSIVE OPERATORS
}

\author{
GIDEON SCHECHTMAN ${ }^{1}$
}

\begin{abstract}
For each $n, 1<n<\infty$, there exist a weakly compact, convex subset $\boldsymbol{W}$ of $L_{1}$ and a family $\left\{T_{i}\right\}_{i=1}^{n}\left(\left\{T_{i}\right\}_{i=1}^{\infty}\right.$ in case $\left.n=\infty\right)$ of nonexpansive operators of $W$ into $W$ such that there is no common fixed point for the whole family but any (finite) strict subfamily admits a common fixed point.
\end{abstract}

Recently, Alspach [1] found an example of a weakly compact, convex subset of $L_{1}(0,1)$ and a nonexpansive operator on it with no fixed points, thus solving a long-standing problem. Using the basic idea of Alspach we produce in this note a class of such examples which are somewhat simpler than Alspach's and give more variety (cf. Lemma 1 and its corollary). We use the construction to also prove the main results of this note, Theorems 1 and 2 below.

I would like to thank Tadek Figiel and Bill Johnson for some helpful discussions.

THEOREM 1. For every $n=1,2, \ldots$ there exist a weakly compact, convex subset $W$ of $L_{1}$ and a family $T_{1}, T_{2}, \ldots, T_{n+1}$ of $n+1$ commuting, nonexpansive operators of $W$ into itself such that any $n$ of them have a common fixed point but $T_{1} \circ T_{2} \circ \ldots \circ T_{n+1}$ does not have a fixed point. In particular, $T_{1}, T_{2}, \ldots, T_{n+1}$ do not have a common fixed point.

THEOREM 2. There exist a weakly compact, convex subset $W$ of $L_{1}$ and a sequence $T_{1}, T_{2}, \ldots$ of commuting nonexpansive operators of $W$ into itself such that any finite number of them have a common fixed point but there is no common fixed point for the entire sequence.

Definition. Let $(S, \mathcal{F}, \mu)$ be a measure space and let $\tau^{-1}: S \rightarrow[0,1] \times S$ be a measure preserving transformation. We define a (nonlinear) operator $T_{\tau}$ on $\{f \in$ $\left.L_{1}(S) ; 0 \leqslant f \leqslant 1\right\}$ by

$$
T_{\tau} f(s)=\chi_{\tau}(\{(t, s) ; 0<t<f(s)\}) .
$$

It is easily checked that $T_{\tau}$ is a (nonlinear) isometry. In particular, $\int T_{\tau} f d \mu=$ $\int f d \mu$ for all $f \in L_{1}(S), 0 \leqslant f \leqslant 1$.

For $0<\alpha \leqslant \beta<1$ let

$$
W_{\alpha, \beta}=\left\{f \in L_{1}(S) ; 0 \leqslant f \leqslant 1, \alpha \leqslant \int f \leqslant \beta\right\} .
$$

$W_{\alpha, \beta}$ is a weakly compact, convex subset of $L_{1}(S)$ and $T_{\tau}\left(W_{\alpha, \beta}\right) \subseteq W_{\alpha, \beta}$.

Received by the editors October 28, 1980 and, in revised form, March 26, 1981.

1980 Mathematics Subject Classification. Primary 47H09, 47H10.

Key words and phrases. Common fixed point, nonexpansive operators, weakly compact sets, convex sets.

${ }^{1}$ Supported in part by NSF Grant No. MCS-79-03042. 
LEMMA 1. Let $0<\alpha<\beta<1$. $T_{\tau}$ has a fixed point in $W_{\alpha, \beta}$ if and only if there exists a set $A \in \mathscr{F}$ with $\alpha \leqslant \mu(A) \leqslant \beta$ and $\tau([0,1] \times A)=A$ a.e. If there exists such a set $A, \chi_{A}$ is a fixed point.

We leave the simple proof to the reader.

Corollary (D. AlsPaCh). There exist a weakly compact, convex subset $W$ of $L_{1}$ and a nonexpansive operator on $W$ with no fixed point.

Proof. Let $W=W_{1 / 2,1 / 2}, S=[0,1]^{\kappa_{0}}$ with the product Lebesgue measure and let $\tau$ be defined by $\tau\left(t,\left(s_{1}, s_{2}, \ldots\right)\right)=\left(t, s_{1}, s_{2}, \ldots\right)$; then $\tau([0,1] \times A)=A$ a.e. if and only if $\mu(A)=0$ or $\mu(A)=1$ so $T_{\tau}$ does not have a fixed point.

(Another example: $S=[0,1]$ with Lebesgue measure

$$
\tau\left(\sum_{n=1}^{\infty} \varepsilon_{n} 2^{-n}, \sum_{n=1}^{\infty} \delta_{n} 2^{-n}\right)=\frac{\varepsilon_{1}}{2}+\frac{\delta_{1}}{4}+\frac{\varepsilon_{2}}{8}+\frac{\delta_{2}}{16}+\cdots,
$$

$\left.\varepsilon_{n}, \delta_{n}=0,1, n=1,2, \ldots\right)$

Let $P_{S}:[0,1] \times S \rightarrow S$ be defined by $P_{S}(t, s)=s$. Given $\tau, \sigma$ such that $\tau^{-1}, \sigma^{-1}$ are measure preserving transformations of $S$ onto $[0,1] \times S$, we define $\pi=\pi(\tau, \sigma)$ by

$$
\pi^{-1}=\sigma^{-1} P_{S} \tau^{-1}
$$

It is easily checked that $\pi^{-1}$ is a measure preserving transformation, considered as a map from $(S, \mathscr{B}, \mu)$ to $[0,1] \times(S, \mathscr{F}, \mu)$ where $\mathscr{B}=\{\tau([0,1] \times B) ; B \in \mathscr{F}\}$.

LEMMA 2. With the notations as above, $T_{\tau} \circ T_{\sigma}=T_{\pi}$.

Proof.

$$
\begin{aligned}
T_{\tau} \circ T_{\sigma} f & =T_{\tau} \chi_{\sigma\{(t, s) ; 0<t<f(s)\}} \\
& =\chi_{\tau}\{[0,1] \times \sigma\{(t, s) ; 0<t<f(s)\}\} \\
& =\chi_{\pi\{(t, s) ; 0<t<f(s)\}}=T_{\pi} f .
\end{aligned}
$$

LEMMA 3. $T_{\tau}$ and $T_{\sigma}$ commute as operators on $\{f ; 0 \leqslant f \leqslant 1\}$ if and only if $\tau([0,1] \times \sigma(B))=\sigma([0,1] \times \tau(B))$ a.e. for all $B \subseteq[0,1] \times S, B$ measurable.

Proof. The if part follows from the proof of the previous lemma. For the only if part notice that if $T_{\tau}$ and $T_{\sigma}$ commute then taking $f=\alpha \cdot \chi_{A}$ in the second line of the proof of Lemma 2, we get $\tau\{[0,1] \times \sigma(B)\}=\sigma\{[0,1] \times \tau(B)\}$ for all $B$ of the form $[0, \alpha] \times A$. From this one easily gets the same equality for all measurable $B$.

From now on we assume $S=[0,1]^{\aleph_{0}}$.

Given a one-to-one measure preserving transformation $\rho:[0,1] \rightarrow[0,1]$, we define $\tau=\tau(\rho):[0,1] \times S \rightarrow S$ by

$$
\tau\left(t,\left(s_{1}, s_{2}, \ldots\right)\right)=\left(\rho\left(s_{1}\right), t, s_{2}, s_{3}, \ldots\right),
$$

and given $k=1,2, \ldots$ we define $\psi^{-1}: S \rightarrow S$ by

$$
\psi\left(s_{1}, s_{2}, \ldots\right)=\rho\left(s_{1}\right) \times[0,1]^{k} \times\left(s_{2}, s_{3}, \ldots\right)
$$

(i.e. $\left.\psi^{-1}\left(s_{1}, s_{2}, \ldots\right)=\left(\rho^{-1}\left(s_{1}\right), s_{k+2}, s_{k+3}, \ldots\right)\right)$. 
LEMMA 4. (1) Let $A \subseteq[0,1]$ be measurable; then $\rho(A)=A$ a.e. if and only if $\tau\left([0,1] \times A \times[0,1]^{\aleph_{0}}\right)=A \times[0,1]^{\kappa_{0}}$ a.e.

(2) If $\rho$ is ergodic (i.e. $\rho(A)=A$ a.e. implies $\mu(A)=0$ or $\mu(A)=1$ ) then $\psi$ is ergodic.

(3) If $\rho_{1}, \rho_{2}$ commute then $T_{\tau\left(\rho_{1}\right)}, T_{\tau\left(\rho_{2}\right)}$ commute.

Proof. (1) $\tau\left([0,1] \times A \times[0,1]^{\kappa_{0}}\right)=\rho(A) \times[0,1]^{\kappa_{0}}$ and $\rho(A) \times[0,1]^{\mu_{0}}=A \times$ $[0,1]^{\kappa_{0}}$ a.e. if and only if $\rho(A)=A$ a.e.

(2) In the language of ergodic theory, $\psi$ is a one-to-many transformation on a product space which is a product of an ergodic transformation with a (one-tomany) mixing transformation. It is a known fact that such a transformation is ergodic. Since books on ergodic theory usually deal only with one-to-one transformations, we reproduce the proof of this fact in our special case.

Since $\psi$ is a measure preserving transformation, the operator $T$, defined on $L_{2}(S)$ by $T f(s)=f\left(\psi^{-1}(s)\right)$, is an isometry. If $f(s)=f_{1}\left(s_{1}\right) \cdot f_{2}\left(s_{2}\right) \cdots \cdot f_{l}\left(s_{l}\right)$ and $g(s)=$ $g_{1}\left(s_{1}\right) \cdot g_{2}\left(s_{2}\right) \cdots \cdot g_{l}\left(s_{l}\right)$, then

$$
T^{i} f=f_{1}\left(\rho^{-i}\left(s_{1}\right)\right) \cdot f_{2}\left(s_{i k+2}\right) \cdot f_{3}\left(s_{i k+3}\right) \cdots \cdot f_{l}\left(s_{i k+l}\right)
$$

and

$$
\begin{aligned}
\lim _{n \rightarrow \infty} \frac{1}{n} & \sum_{i=0}^{n-1}(T \cdot f, g) \\
& =\left(\lim _{n \rightarrow \infty} \frac{1}{n} \sum_{i=0}^{n-1} f_{1} \circ \rho^{-i}, g_{1}\right) \cdot \int f_{2} \cdot \int \bar{g}_{2} \cdot \int f_{3} \cdot \int \bar{g}_{3} \cdots \cdots \int f_{l} \cdot \int \bar{g}_{l} \\
& =\int f_{1} \cdot \int \bar{g}_{1} \cdot \int f_{2} \cdot \int \bar{g}_{2} \ldots \cdot \int f_{l} \cdot \int \bar{g}_{l}=\int f \cdot \int \bar{g} .
\end{aligned}
$$

The limit in the second term is in $L_{2}$; its existence and equality to $\int f_{1}$ follow from the simplest form of the ergodic theorem (cf. [2]). It follows that for any $f, g \in L_{2}$,

$$
\lim _{n \rightarrow \infty} \frac{1}{n} \sum_{i=0}^{n-1}\left(T^{i} f, g\right)=\int f \cdot \int \bar{g} .
$$

In particular, if $A$ is such that $\psi(A)=A$ then $T^{i} \chi_{A}=\chi_{A}$ for all $i$ and

$$
\left(\chi_{A}, g\right)=\mu(A) \cdot \int \bar{g} \text { for all } g \in L_{2} \text {. }
$$

For $g=\chi_{1}$ we get $\mu(A)=\mu(A)^{2}$ so $\mu(A)=0$ or $\mu(A)=1$.

(3) is easily checked using Lemma 3.

Proof of Theorem 2. $W=W_{1 / 2,1 / 2}$. Let $\left\{r_{i}\right\}_{i=1}^{\infty}$ be an enumeration of the rationals in $[0,1]$, let $\rho_{i}$ be defined by $\rho_{i}(t)=t+r_{i}(\bmod 1)$ and let $T_{i}=T_{\tau\left(\rho_{1}\right)}$. By Lemma $4.3\left\{T_{i}\right\}_{i=1}^{\infty}$ commute. Given $n$ let $m$ be a common denominator for $r_{1}, \ldots, r_{n}$ and let $A=\cup_{k=0}^{m-1}(k / m, k / m+1 / 2 m)$; then $\mu(A)=\frac{1}{2}$, and $\rho_{i}(A)=A$ for $i=1, \ldots, n$. By Lemmas 1 and $4.1 \chi_{1}$ is a common fixed point for $T_{1}, \ldots, T_{n}$. If $\chi_{1}$ is a fixed point for all the $T_{i}$ 's, then $\tau\left(\rho_{i}\right)([0,1] \times A)=A$ for $i=1,2, \ldots$. It follows easily that $\tau(\rho)([0,1] \times A)=A$ for any $\rho$ of the form $\rho(t)=t+\alpha(\bmod 1), \alpha \in[0,1]$. However, if $\alpha$ is irrational, $\rho$ is ergodic in contradiction to Lemma 4.2 (with $k=1$ ). 
Before starting the proof of Theorem 1 let us illustrate the idea by looking at the case $n=1$. Divide the interval $[0,1]$ into six successive intervals $I_{1}, I_{2}, \ldots, I_{6}$ each of length $\frac{1}{6}$. Let $\varepsilon:[0,1] \rightarrow[0,1]$ be a measure preserving transformation such that $\varepsilon\left(I_{i}\right)=I_{i}, i=1, \ldots, 6$, and $\varepsilon^{12}$ is ergodic relative to each of the $I_{i}$, i.e. $I_{i}$, $i=1, \ldots, 6$, and their finite unions are the only nontrivial $\varepsilon^{12}$-invariant sets. Let $\rho_{1}$ be the transformation which carries $I_{i}$ linearly onto $I_{i+2}(\operatorname{Mod} \sigma)$ followed by $\varepsilon$, and let $\rho_{2}$ be the transformation which switches $I_{1}$ with $I_{2}, I_{3}$ with $I_{4}, I_{5}$ with $I_{6}$ followed by $\varepsilon$. Clearly, $I_{1} \cup I_{3} \cup I_{5}$ is invariant under $\rho_{1}$ while $I_{1} \cup I_{2}$ is invariant under $\rho_{2}$. The composition $\rho_{1} \circ \rho_{2}=\rho_{2} \circ \rho_{1}$ is the transformation which carries linearly $I_{1} \rightarrow I_{4} \rightarrow I_{5} \rightarrow I_{2} \rightarrow I_{3} \rightarrow I_{6} \rightarrow I_{1}$ followed by $\varepsilon^{12}$ and it is easily seen to be ergodic. If we now define $T_{i}=T_{\tau\left(\rho_{i}\right)}, i=1,2$, we get the desired result for $n=1$.

Proof of Theorem 1. Fix $n$, let $p_{1}, \ldots, p_{n+1}$ be the first $n+1$ prime numbers $\left(p_{1}=2\right)$ and let $p=p_{1} p_{2} \cdots p_{n+1}$. Let $\varepsilon:\left[0, p^{-1}\right) \rightarrow\left[0, p^{-1}\right)$ be a one-to-one measure preserving transformation with $\varepsilon^{k}$ ergodic for all $k=1,2, \ldots$ For $i=1,2, \ldots, n+1$ define a measure preserving transformation $\rho_{i}$ of $[0,1)$ onto itself by

$$
\rho_{i}(x)=\left(\varepsilon\left(x-k p^{-1}\right)+k p^{-1}+p_{i}^{-1}\right)(\bmod 1) \text { if } k p^{-1}<x<(k+1) p^{-1} .
$$

Let $\bar{\tau}_{i}=\tau\left(\rho_{i}\right)$ and $\bar{T}_{i}=T_{\bar{\tau}_{i}}$.

Given any subset of $\left\{\bar{T}_{i}\right\}_{i=1}^{n+1}$ of cardinality $n$, say $\left\{\bar{T}_{i}\right\}_{i=1, i \neq j}^{n+1}$, let $A=$ $\cup_{k=0}^{p p_{j}^{-1}-1}\left[k p_{j} p^{-1}, k p_{j} p^{-1}+p^{-1}\right)$; then $\mu(A)=p_{j}^{-1}$ and $\rho_{i}(A)=A$ for all $i \neq j$. It follows from Lemmas 1 and 4.1 that $\chi_{A \times[0,1]^{\omega_{0}}}$ is a fixed point of $\bar{T}_{i}$ for all $i \neq j$.

By the Chinese Remainder Theorem there are positive integers $a_{1}, a_{2}, \ldots, a_{n+1}$ such that $p^{-1}=\sum_{i=1}^{n+1} a_{i} p_{i}^{-1}(\bmod 1)$. Put $T_{i}=\left(\bar{T}_{i}\right)^{a_{i}}, i=1, \ldots, n+1$. We claim that $\left\{T_{i}\right\}_{i=1}^{n+1}$ and $W=W_{\alpha, \beta}$, where $\alpha=\min _{1<i<n+1} p_{i}^{-1}, \beta=\frac{1}{2}$, satisfy the conclusion of Theorem 1 .

From the first part of the proof it is clear that any $n$ of $T_{1}, \ldots, T_{n+1}$ have a common fixed point. Since $\rho_{1}, \ldots, \rho_{n+1}$ commute, $\bar{T}_{1}, \ldots, \bar{T}_{n+1}$ and, thus, $T_{1}, \ldots, T_{n+1}$ commute. To show that $T=T_{1} \circ \cdots \circ T_{n+1}$ does not have a fixed point notice that, by Lemma $2, T=T_{\psi}$ where

$$
\psi\left(t,\left(s_{1}, s_{2}, \ldots\right)\right)=\left(\rho_{1}^{a_{1}} \circ \ldots \circ \rho_{n+1}^{a_{n+1}}\right)\left(s_{1}\right) \times[0,1]^{a} \times\left(t, s_{2}, s_{3}, \ldots\right),
$$

$a=a_{1}+a_{2}+\cdots+a_{n+1}$. By Lemmas 1 and 4.2 it is enough to show that $\rho=\rho_{1}^{a_{1}} \circ \ldots \circ \rho_{n+1}^{a_{n+1}}$ is ergodic, but $\rho(x)=\left(\varepsilon^{a}\left(x-k p^{-1}\right)+(k+1) p^{-1}\right)(\bmod 1)$ if $k p^{-1} \leqslant x<(k+1) p^{-1}$, and the ergodicity of $\rho$ follows easily from that of $\varepsilon^{a}$.

\section{REFERENCES}

1. D. E. Alspach, A fixed point free nonexpansive map, Proc. Amer. Math. Soc. 82 (1981), 423-424.

2. N. Dunford and J. T. Schwartz, Linear operators. I, Interscience, New York, 1958.

Department of Mathematics, Ohio State University, Columbus, Ohio 43210

Current address: Department of Theoretical Mathematics, The Weizmann Institute of Science, Rehovot, Israel 